\title{
Casuística de pacientes asistidos en una policlínica de hipertensión arterial
}

\author{
Dres. María Victoria Toledo, Paola Spósito, Mario Llorens
}

\section{Resumen}

Introducción: la hipertensión arterial (HTA) es uno de los factores de riesgo cardiovascular más prevalentes en el mundo. La detección temprana, el tratamiento apropiado y su control determinan importantes beneficios en materia de salud y economía. Dada la alta incidencia de consultas por complicaciones vinculadas a esta enfermedad, se crea en el año 2014 la policlínica de HTA en el Hospital Maciel, realizándose en ella tratamiento integral de pacientes hipertensos, fundamentalmente de aquellos de difícil control.

Objetivo: caracterizar la población de hipertensos que asisten a esta policlínica, así como mostrar los resultados de su implementación respecto al control de las cifras tensionales.

Material y método: estudio descriptivo, longitudinal, retrospectivo y prospectivo entre los años 2015 y 2017 que incluyó a los pacientes que se asisten en dicha policlínica, excluyendo a embarazadas, menores de 18 años, pacientes que no se controlaron en el último año y fallecidos.

Resultados: se analizó un total de 122 pacientes, media de edad 59,8 años, predominio sexo femenino (1,8:1). Factores de riesgo cardiovascular asociados: sobrepeso/obesidad (88\%), dislipemia (65\%), tabaquismo (39\%) y diabetes (29\%). Prevalencia de daño de órgano blanco en el momento de ser derivados, $62 \%$. El 98\% recibe tratamiento farmacológico: $71 \%$ terapia combinada. Predomina la HTA grado III $(68,9 \%)$.

Conclusión: la población se caracteriza por una elevada incidencia de sobrepeso/obesidad, HTA grado III, y alta prevalencia de daño de órgano blanco en el momento de la consulta, lo cual conduce a pensar que los pacientes se derivan tardíamente. En el subgrupo de hipertensos grado III se logró un adecuado control promedio de cifras tensionales en el seguimiento anual.

Palabras clave: HIPERTENSIÓN

FACTORES DE RIESGO

PATRONES DE VARIABILIDAD

\section{Casuistry of patients attended in a polyclinic of arterial hypertension}

\section{Summary}

Introduction: high blood pressure is one of the most prevalent cardiovascular risk factors in the world. Early detection, appropriate treatment and control determine important health and economic benefits. Given the high incidence of consultations due to complications of this disease, in 2014 the hypertension polyclinic was created in the Maciel Hospital, in which evaluation and treatment of the hypertensive patient were carried out in an integral way, fundamentally for those with difficult control.

Objective: to characterize the population of hypertensive patients attending this polyclinic, as well as to show the results of its implementation regarding the figures control.

Materials and methods: descriptive, longitudinal, retro and prospective study between 2015-2017 that includes patients who are attended by polyclinic, excludes pregnant women, young persons under 18 years old, those who were not controlled in the last year and deceased.

Results: 122 patients were analysed, average age 59,8 years old, female sex 1.8:1. Associated cardiovascular risk factors: overweight/obesity (88\%), dyslipidemia (65\%), smoking (39\%) and diabetes (29\%). Prevalence of white organ damage $62 \%$ at the time of referral. $98 \%$ receive pharmacological treatment: $71 \%$ combined therapy. Hypertension grade III predominates $(68,9 \%)$.

Clínica Médica 3, Hospital Maciel, Facultad de Medicina, Universidad de la República. Montevideo, Uruguay.

Correspondencia: Dra. María Victoria Toledo. Correo electrónico: vickytoledopereda@gmail.com

Los autores declaran no tener conflictos de intereses ni fuentes de financiamiento.

Por la naturaleza del estudio y la preservación del anonimato de los pacientes no se consideró necesario someterlo a juicio del Comité de Ética de la Institución.

Recibido Set 7, 2018; aceptado Jun 12, 2019 
Conclusion: the population is characterized by a high incidence of overweight/obesity, hypertension grade III as well as high prevalence of white organ damage at the time of consultation, which leads to think that the patients are derived late. In the subgroup of hypertensives grade III adequate average control of blood pressure figures was achieved in the annual follow-up.

Key words:

HYPERTENSION

RISK FACTORS

PATTERNS OF VARIABILITY

\section{Casuística de pacientes atendidos na policlínica de hipertensão arterial}

\section{Resumo}

Introdução: a hipertensão arterial é um dos fatores de risco cardiovascular mais prevalentes no mundo. A detecção precoce, o tratamento e o controle apropriados determinam importantes benefícios econômicos e para a saúde. Dada a alta incidência de consultas relacionadas a complicações desta doença, foi criado em 2014 a policlínica de hipertensão em Hospital Maciel, realizando avaliação e tratamento de pacientes hipertensos de forma holística, principalmente daqueles de difícil controle.

Objetivo: caracterizar a população de hipertensos atendidos nesta policlínica, bem como mostrar os resultados de sua implementação no controle de figuras.

Materiais e métodos: estudo descritivo, longitudinal, retro e prospectivo, entre os anos de 2015 e 2017, que incluiu os pacientes que compareceram à policlínica, excluindo gestantes, menores de 18 anos, pacientes que não foram controlados no último ano e falecidos.

Resultados: foram analisados 122 pacientes, idade média de 59,8 anos, sexo feminino 1,8: 1. Fatores de risco cardiovascular associados: sobrepeso/obesidade (88\%), dislipidemia (65\%), tabagismo (39\%) e diabetes (29\%). Prevalência de lesão de órgão branco $62 \%$ no momento do encaminhamento. $98 \%$ recebem tratamento farmacológico: $71 \%$ terapia combinada. Hipertensão grau III predomina (68,9\%).

Conclusão: a população é caracterizada por uma alta incidência de sobrepeso / obesidade, hipertensão grau III, bem como a alta prevalência de lesão de órgão branco no momento da consulta, o que leva a pensar que os pacientes são derivados tardiamente. No subgrupo de hipertensos grau III, o controle médio adequado dos valores da pressão arterial foi alcançado no seguimento anual.

Palavras-chave: HIPERTENSÃO

FACTORES DE RISCO

PADRÕES DE VARIABILIDADE

\section{Introducción}

La hipertensión arterial (HTA) es uno de los factores de riesgo cardiovascular (CV) de mayor prevalencia en el mundo. La detección temprana, el tratamiento apropiado y el control de la misma determinan importantes beneficios para la salud pública, ya que el tratamiento de las complicaciones vinculadas a esta enfermedad abarca intervenciones costosas que aumentan los presupuestos estatales e individuales ${ }^{(1)}$

A nivel mundial las estadísticas informan que en el año 2008 aproximadamente $40 \%$ de los adultos mayores de 25 años fueron diagnosticados como hipertensos $^{(2)}$.

En nuestro país, en 2016, Sandoya y colaboradores realizaron un estudio de corte transversal mediante revisión retrospectiva de historias clínicas que incluyó 2.020 pacientes, 1.134 provenientes de instituciones públicas y 886 de instituciones privadas asistidos en policlínicas desde el 1 de agosto del
¿Qué aporta este estudio al conocimiento actual?

El presente estudio permite caracterizar una población de pacientes hipertensos que se asisten en una policlínica de referencia, poniendo de manifiesto el beneficio de una atención individualizada e interdisciplinaria. Conocer las características de la población nos permitirá realizar cambios, planificando mejorar una estrategia de diagnóstico precoz y terapéutica dirigida a esta población.

2013 al 31 de julio del 2015 con el objetivo de estimar la prevalencia y el manejo de la hipertensión de acuerdo al nivel socioeconómico. Encontraron mayor prevalencia de hipertensos en quienes se atienden a nivel público, $64 \%$ vs $50 \%$ a nivel privado ( $p<0,05$ ). Concluyen que el cuidado que se brinda a los pacientes en salud pública es de menor calidad, encontrando mayor porcentaje de normalización de las cifras de 
presión arterial (PA) entre quienes se atienden a nivel privado ${ }^{(3)}$.

Dada la alta incidencia de HTA en la Administración de los Servicios de Salud del Estado (ASSE), se crea en 2014 la policlínica de HTA integrada por equipo docente, residentes de Clínica Médica 3 y cardiólogos del Hospital Maciel. El objetivo es valorar al paciente de una manera integral, enfocados en la prevención y diagnóstico precoz del daño de órgano blanco (DOB) haciendo hincapié en aquellos pacientes de difícil manejo, con el fin de brindar una mejor atención a los usuarios.

El objetivo de este estudio es caracterizar la población de hipertensos que concurre a la policlínica de HTA del Hospital Maciel y evaluar el control de la enfermedad en el subgrupo de hipertensos grado III.

\section{Material y método}

Se incluyeron todos los pacientes que asistieron a la policlínica de HTA en el período comprendido entre el 2 de marzo de 2015 y el 31 de octubre del 2017 inclusive, siendo éstos derivados por médicos generales, internistas o cardiólogos, por mal control de cifras de PA o por complicaciones vinculadas a esta enfermedad. Se excluyeron embarazadas, menores de 18 años, los que no concurrieron a control en el último año y los fallecidos. El total de pacientes incluidos fue de 122 .

Los datos se recogieron del análisis de historias clínicas donde se ingresan los antecedentes personales, estudios para valoración de DOB y el control de cifras de PA, no pudiendo determinar en ellas el cumplimiento del tratamiento farmacológico, ni del régimen hiposódico, datos que se obtuvieron a través del interrogatorio de los pacientes.

Todos los pacientes que ingresan a la policlínica, salvo excepciones que se detallarán, son evaluados mediante monitoreo ambulatorio de presión arterial (MAPA) cuyo resultado permite en la mayor parte de los casos confirmar el diagnóstico de HTA y determinar el patrón de variabilidad y severidad de ésta. No se dispuso de manguitos adecuados al diámetro del miembro superior de algunos pacientes obesos, por lo cual no se pudo realizar el registro, quedando un total de 82 pacientes con MAPA efectuado. En los 40 pacientes restantes sin MAPA, se utilizó el monitoreo domiciliario de la PA (MDPA) como técnica de control. Se instruyó a todos los pacientes entregándose un folleto explicativo sobre cómo se realiza una medición adecuada de la PA en domicilio y efectuando un entrenamiento previo en el consultorio. Se utilizaron aparatos digitales OMRON para medición en arteria humeral. Las mediciones se realizaron en la parte inferior del brazo con el manguito adaptado al perímetro de éste y en contacto con la piel, con el puño a nivel del corazón y el paciente sentado en forma adecuada, con la espalda apoyada en el respaldo y en un ambiente tranquilo. A los pacientes con valores de PA no controlada se les realizó seguimiento cada 15 días para evitar la inercia terapéutica hasta lograr controles adecuados. Los pacientes con valores óptimos se controlaron cada tres meses.

A cada paciente que ingresa a la policlínica se le solicita en la consulta inicial los estudios correspondientes para valoración de DOB:

Electrocardiograma (ECG): para evaluar hipertrofia ventricular izquierda (HVI), evidenciada por índice de Sokolow-Lyon > 3,5 mV, $\mathrm{R}$ aVL $>1,1 \mathrm{mV}$, índice de Cornell > $2.440 \mathrm{~mm} . \mathrm{ms}$.

Ecocardiograma transtorácico (ETT) considerando a esta técnica más sensible que el ECG para el diagnóstico de HVI.

Creatininemia, filtrado glomerular por fórmula de la Chronic Kidney Disease EPIdemiology Collaboration (CKD-EPI) y albuminuria en muestra de orina aislada para diagnosticar enfermedad renal crónica.

Oftalmoscopía para detectar retinopatía hipertensiva.

Test de evaluación de daño cognitivo: MOCA/mínimo examen cognitivo.

Tomografía computarizada de cráneo: para descartar daño encefálico.

Se consideró enfermedad CV establecida, de acuerdo a las Guías de práctica clínica The Task Force for the management of arterial hypertension of the European Society of Cardiology (ESC) and the European Society of Hypertension (ESH) 2018 para el manejo de la HTA la presencia de: accidente cerebrovascular isquémico o hemorrágico, ataque isquémico transitorio, cardiopatía isquémica, insuficiencia cardíaca y enfermedad arterial periférica sintomática en extremidades inferiores.

Se consideró diabético al paciente que presentó glucemia casual medida en plasma venoso $\geq 200$ $\mathrm{mg} / \mathrm{dl}$ o glucemia de ayuno medida en plasma venoso $\geq 126 \mathrm{mg} / \mathrm{dl}$ o una prueba de tolerancia oral a la glucosa positiva o hemoglobina glicosilada $>6,5 \%$. Se consideró dislipémicos a los que presentaron colesterol total $>200 \mathrm{mg} / \mathrm{dl}$ o triglicéridos $>150 \mathrm{mg} / \mathrm{dl}$ en al menos un control y se consideró tabaquista a la persona que fumó por lo menos un cigarrillo en los últimos seis meses. Por último, se consideró sobrepeso al índice de masa corporal (IMC) $>25 \mathrm{~kg} / \mathrm{m}^{2} \mathrm{y}$ obesidad al IMC $>30 \mathrm{~kg} / \mathrm{m}^{2}$. 


\begin{tabular}{|c|c|c|c|}
\hline Categoría & $\begin{array}{l}\text { Sistólica } \\
(m m H g)\end{array}$ & & $\begin{array}{c}\text { Diastólica } \\
(m m H g)\end{array}$ \\
\hline PA normal & $<130$ & $\mathrm{y} / \mathrm{o}$ & $<85$ \\
\hline PA normal alta & $130-139$ & $\mathrm{y} / \mathrm{o}$ & $85-89$ \\
\hline HTA grado I & $140-159$ & $\mathrm{y} / \mathrm{o}$ & $90-99$ \\
\hline HTA grado II & $160-179$ & $\mathrm{y} / \mathrm{o}$ & $100-109$ \\
\hline HTA grado III & $\geq 180$ & $\mathrm{y} / \mathrm{o}$ & $\geq 110$ \\
\hline
\end{tabular}

\section{Métodos estadísticos}

Se presentan tablas y gráficos de frecuencias para la descripción de variables cualitativas. En el caso de las continuas se muestran medidas de resumen. En la búsqueda de asociación entre variables se utilizó el test de chi cuadrado o exacto de Fisher en los casos necesarios. Para el estudio de diferencias entre variables cuantitativas se utilizó el test t de Student para muestras independientes y dependientes según requerimiento. El cálculo de prevalencia se realizó teniendo en cuenta el total de casos observados en el total de casos en riesgo. En todos los casos se fijó como nivel de significación un alfa de 0,05.

\section{Definición de variables}

HTA: PA sistólica (PAS) $\geq 140$ mmHg o PA diastólica $(\mathrm{PAD}) \geq 90 \mathrm{mmHg}$, según la evidencia derivada de ensayos clínicos aleatorizados que indica que en pacientes con estos valores de PA las reducciones inducidas por tratamiento farmacológico son beneficiosas $^{(4)}$.

La clasificación de PA y grados de HTA según la ESC 2018 se muestran en la tabla 1.

Patrón dipper: patrón normal y fisiológico. Se define como un descenso de la PA media nocturna entre $10 \%$ y $20 \%$ con respecto a la PA media diurna. Es el patrón más frecuente en hipertensos no complicados $^{(5)}$.

Patrón non dipper: descenso atenuado de la PA media nocturna entre $0 \%$ y $10 \%$ con respecto a la PA media diurna ${ }^{(5)}$.

Patrón dipper extremo: descenso de la PA media nocturna $>20 \%$ con respecto a la PA media diur$\mathrm{na}^{(5)}$.

Patrón riser: aumento de la PA media nocturna a valores superiores a la media diurna ${ }^{(5)}$.

MAPA: es el monitoreo ambulatorio de la PA mediante un tensiómetro portátil que recoge información durante 24 horas. Su lectura evidencia la variabilidad de la PA diurna y nocturna durante las diferentes actividades que realiza el paciente ${ }^{(4)}$.

Consideramos PA inicial al valor que se constata en la primera consulta, debiendo destacar que todos los pacientes son derivados por médicos generales, internistas y cardiólogos, por ende todos se encontraban bajo tratamiento farmacológico.

PA sistólica media: promedio de valores de la PAS al ingreso de todos los pacientes.

PA diastólica media: promedio de valores de PAD al ingreso de todos los pacientes.

\section{Resultados}

\section{Población total de hipertensos}

Asistieron a la policlínica de HTA en el período previamente mencionado 130 pacientes, siendo la población final de 122 pacientes según criterios de inclusión y exclusión, 82 con MAPA realizado a la fecha del comienzo del estudio y 40 con MDPA. Se observó predominio del sexo femenino, 64,8\% (79 pacientes), existiendo una razón de 1,8 mujeres/hombres. El promedio de edad correspondió a 59,6 4 1,3 años, con un mínimo y un máximo de 19 y 88 años, respectivamente. En el caso de los pacientes de sexo masculino, correspondió a 58,9 $\pm 2,2$ años, mientras que en el caso de las mujeres fue de 59,9 $\pm 1,6$ años, no existiendo diferencias estadísticamente significativas para la edad según sexo, $\mathrm{p}=0,716$.

Los factores de riesgo CV más frecuentes asociados a la HTA (figura 1) fueron sobrepeso/obesidad $87,7 \%$ (107 pacientes) y dislipemia 64,8\% (79 pacientes), seguidos en menor porcentaje por tabaquismo $39,3 \%$ (48 pacientes) y DM 28,7\% (35 pacientes).

La prevalencia de DOB fue de $62,3 \%$ (76 pacientes).

En relación con el grado de HTA, 68,9\% (84 pacientes) presenta grado III (figura 2). El 67,2\% (82 pacientes) tiene MAPA realizado a la fecha (por lo expuesto previamente), siendo en éstos el patrón de variabilidad más frecuente el non dipper en 46,3\% (38 pacientes) (figura 3). En los pacientes controlados con MDPA no contamos con el patrón de variabilidad correspondiente.

La mayoría de los pacientes recibe tratamiento farmacológico: 98,4\% (120 pacientes), y 70,8\% de ellos (85 pacientes) cumple con el mismo. El 70,8\% (85 pacientes) recibe terapia combinada y el $29,2 \%$ (35 pacientes) monoterapia.

En la tabla 2 se presentan las características generales de la población en estudio. 


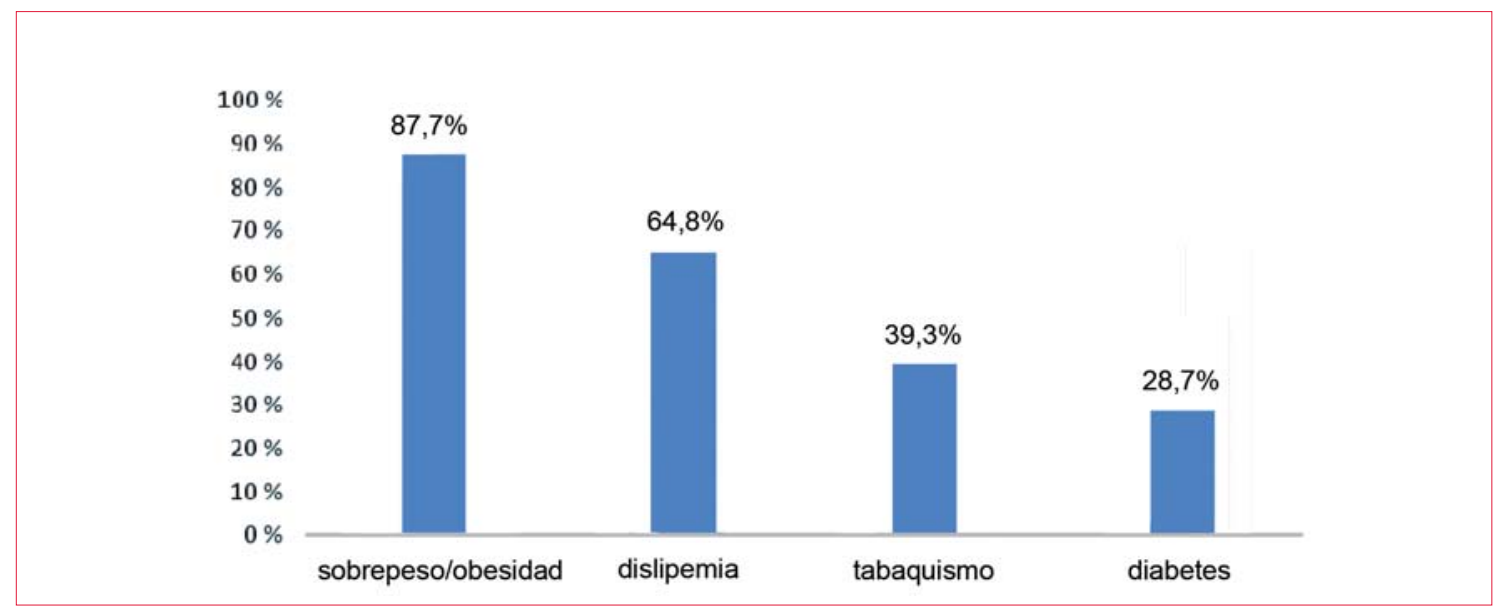

Figura 1. Factores de riesgo cardiovascular más frecuentemente asociados a hipertensión arterial.

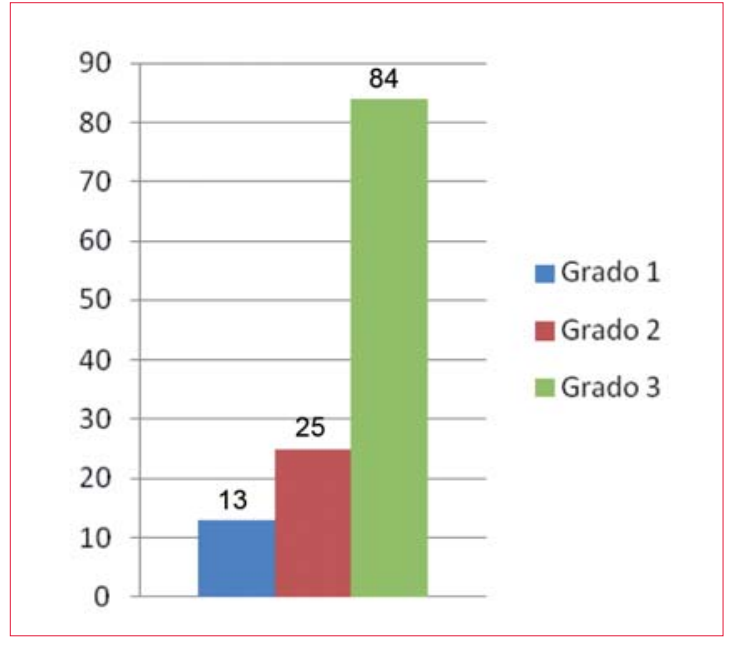

Figura 2. Grados de hipertensión arterial (números absolutos).

\section{Subgrupo de 84 pacientes con hipertensión arterial grado III}

Se caracterizan por presentar sobrepeso/obesidad $88,1 \%$ (74 pacientes), dislipemia $64,3 \%$ (54 pacientes), DM 33,3\% (28 pacientes) y consumo de tabaco 40,5\% (34 pacientes). Entre los pacientes que tienen MAPA realizado en este subgrupo, el patrón de variabilidad más asociado es el non dipper, $57,1 \%$. La mayoría de los pacientes recibe terapia combinada, $76,2 \%(64 / 84)$.

En el análisis univariado se encontró una asociación estadísticamente significativa entre el patrón non dipper y el grupo con grado III de HTA (OR 4,22 IC95\%, 1,46-12,18, p=0,006) en comparación con los pacientes en grado I-II. En los pacientes con grado III se logró un adecuado control de cifras, considerando buen control los valores normales para la edad, $\leq 130 / 80 \mathrm{mmHg}$ en la mayoría de los pacientes y hasta $139 / 89 \mathrm{mmHg}$ en los mayores de 65 años,se-

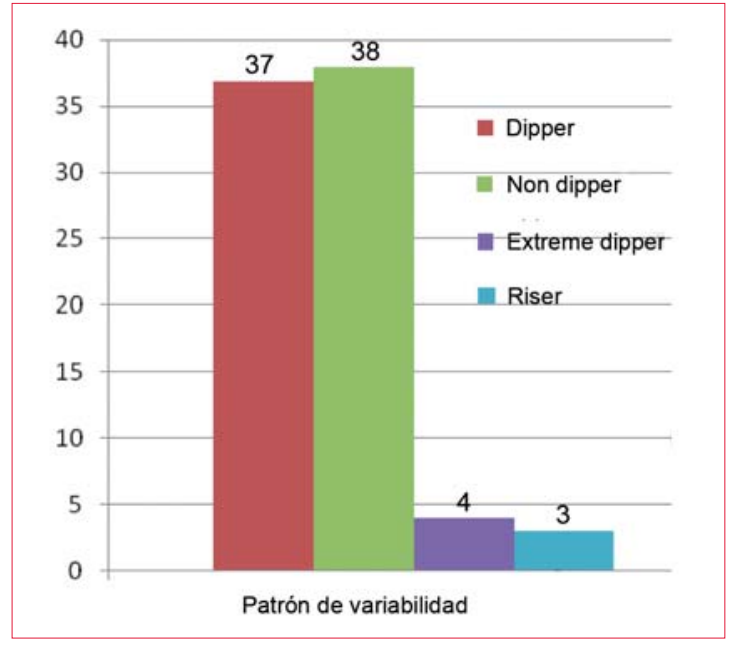

Figura 3. Patrón de variabilidad en 82 pacientes con MAPA realizado (números absolutos).

gún la ESC 2018, realizándose el diagnóstico mediante MAPA. El valor promedio de PAS inicial (en la población con HTA grado III) fue de $156,6 \mathrm{mmHg}$ y al año de seguimiento fue de $130,2 \mathrm{mmHg}$. El valor promedio de PAD inicial fue de $93,3 \mathrm{mmHg}$ y al año disminuyó a 76,9 mmHg.

\section{Discusión}

La HTA es uno de los factores de riesgo más importantes a la hora de analizar el riesgo de muerte por causa CV a diez años, existiendo otros factores que influyen en el pronóstico como DM, ERC, tabaquismo, edad, sexo, dislipemia, obesidad abdominal y el IMC elevado, entre otros ${ }^{(4)}$.

En nuestra policlínica se observó predominio de sexo femenino (relación mujeres/hombres 1,8/1), edad media 59,8 $\pm 1,3$ años sin diferencias significativas de edad con respecto al grupo masculino. Los factores de riesgo $\mathrm{CV}$ presentes fueron en orden de 
Tabla 2. Características de la población estudiada $(n=122)$.

$n(\%)$ o media $\pm D S$

\section{Edad (años)}

Sexo femenino

Sobrepeso/obesidad

Dislipemia

Tabaquismo

Diabetes mellitus

Daño de órgano blanco

MAPA realizado a la fecha

HTA grado III

Tratamiento farmacológico

MAPA: monitoreo ambulatorio de presión arterial; HTA: hipertensión arterial.

frecuencia: sobrepeso/obesidad, dislipemia, tabaquismo y DM. Existe una importante prevalencia de DOB desde el ingreso. El 68,9\% se presentó con HTA grado III. Los datos obtenidos del MAPA evidenciaron predominio del patrón non dipper existiendo una asociación estadísticamente significativa con los grados de HTA (mayor frecuencia en HTA grado III). El tratamiento farmacológico se realizó con terapia combinada en $70,8 \%$, mientras que el resto recibió monoterapia, considerando un adecuado control de cifras evidenciado por MAPA.

La asociación entre HTA y peso ha sido ampliamente documentada. Estudios realizados en Estados Unidos (National Health and Nutrition Examination Survey: NHANES II) demuestran que la prevalencia de HTA en los individuos con sobrepeso u obesos es 2,9 veces mayor que en los de peso nor$\mathrm{mal}^{(6)}$. Es igualmente conocida la asociación entre HTA, DM y dislipemia. Garrido y colaboradores, en un estudio chileno, encontraron la coexistencia de DM y dislipemia en 38,5\% y 57\% de los pacientes hipertensos, respectivamente, porcentajes similares a los hallados en este estudio ${ }^{(7,8)}$.

No se sabe con exactitud la razón de la asociación HTA/DM, pero se ve reflejada en diferentes estudios, destacando la complejidad de lograr un adecuado control de ambos factores de riesgo cuando son concomitantes ${ }^{(8,10)}$. El estudio CARMELA evidenció que en pacientes diabéticos la prevalencia de HTA es 1,5-3 veces mayor que en los no diabéti$\cos ^{(9)}$.

Cabe destacar la importancia que ha adquirido en la última década el conocimiento del patrón de variabilidad de la HTA y su relación con el riesgo cardio y cerebrovascular. El grupo non dipper se caracteriza por asociarse a mayor DOB y peor pronóstico respecto al patrón dipper ${ }^{(10)}$. Estudios revelan que la "cronoterapia" (evaluación del patrón circadiano de la PA mediante MAPA y ajuste del tratamiento medicamentoso) evidencia una correlación directa con la reducción de la PA nocturna, con la modificación de patrón non dipper a dipper, y con un mejor control de la PA ambulatoria de 24 horas. Actualmente se considera que la HTA nocturna es un parámetro más sensible para medir riesgo $\mathrm{CV}$ y el beneficio que tiene el tratamiento nocturno es bien conocido en pacientes con sobrepeso y patrón non dipper, formando parte de este grupo la mayoría de los pacientes que se asisten en la policlíni$\mathrm{ca}^{(8,11)}$.

Respecto al tratamiento de la HTA, el 98\% recibe tratamiento farmacológico.

El tratamiento medicamentoso, cualquiera sea, reduce el riesgo de eventos cardiovasculares mayores totales, y las mayores reducciones en la PA producen mayores reducciones en el riesgo ${ }^{(12,13)}$. Los pacientes que concurren a nuestra policlínica son referidos por médicos generales, internistas y cardiólogos, quienes derivan principalmente aquellos de difícil control, coincidiendo con el hecho de que la mayoría de la población, el 69\%, corresponde a hipertensos grado III y presenta un patrón de variabilidad non dipper con el consiguiente mayor riesgo CV.

Otro dato de suma importancia es el hecho de que al momento de la consulta, el 62\% de los pacientes ya presenta DOB, pudiendo presumirse que fueron derivados tardíamente.

\section{Limitaciones}

Las limitaciones estuvieron vinculadas a la metodología, por ser un estudio descriptivo retrospectivo no fue posible obtener todos los datos para el análisis de algunas de las variables analizadas, como el DOB.

Asimismo, se utilizaron para el análisis cifras promedio de PA iniciales y finales de la cohorte y no los valores de cada uno de los pacientes durante el seguimiento, por lo que podría ocurrir que algunos individuos no hubieran alcanzado cifras tensionales óptimas.

\section{Conclusiones}

La población de hipertensos asistida en nuestra policlínica se caracteriza por tener otros factores de riesgo CV asociados en porcentaje similar al encontrado en estudios internacionales, predominando la obesidad y la dislipemia. 
La mayoría de los pacientes tiene HTA grado III presentando una asociación estadísticamente significativa con el patrón non dipper. En este subgrupo se logró un adecuado control de cifras mediante terapia combinada.

Cabe destacar la gran incidencia de sobrepeso/obesidad, así como una elevada prevalencia de DOB al momento de la consulta, por lo que se podría pensar que estos pacientes son derivados en forma tardía.

Conocer la casuística de nuestra población nos permitirá en el futuro abordar la implementación de estrategias de diagnóstico y evaluación más completas e individualizadas a cargo de un equipo multidisciplinario con el firme objetivo de disminuir la morbimortalidad que genera esta enfermedad.

Contribución de autores

María Victoria Toledo,

https://orcid.org/0000-0002-3175-9955, recolección de datos, redacción, tablas, correcciones y respuesta a editores.

Paola Spósito, https://orcid.org/0000-0001-8715-8385, supervisión del trabajo, redacción y corrección final.

Mario Llorens, https://orcid.org/0000-0002-8762-1592, corrección final.

\section{Bibliografía}

1. World Health Organization. A global brief on hypertension: silent killer, global public health crisis [Internet]. Geneva: WHO; 2013 [consulta 31 Ago 2014]. Disponible en: http://www.who.int/cardiovascular_diseases/publications/global_brief_hypertension/en/

2. Organización Mundial de la Salud. Informe sobre la situación mundial de las enfermedades no transmisibles 2010. Resumen de orientación [Internet]. Ginebra: OMS; 2011[consulta 27 May 2019]. Disponible en: https://www.who.int/nmh/publications/ ncd_report_summary_es.pdf

3. Sandoya E, Torres F, Rivedieu F, Machado F, Nuñez E. Situación de la hipertensión arterial a nivel público y privado. Rev Urug Cardiol. 2016; 31(2): 219-27

4. Williams B, Mancia G, Spiering W, Agabiti Rosei E, Azizi M; Burnier M, et al. $2018 \mathrm{ESC} / \mathrm{ESH}$ guidelines for the management of arterial hyperten- sion. Eur Heart J. 2018; 39, 3021-104. doi: 10.1093/ eurheartj/ehy339

5. Gorostidi M, Segura J, de la Sierra A. Hipertensión arterial nocturna. Hipertens Riesgo Vasc. 2010; 27 Supl 1:S26-33.

6. Nguyen NT, Magno CP, Lane KT, Hinojosa MW, Lane JS. Association of hypertension, diabetes, dyslipidemia, and metabolic syndrome with obesity: findings from the National Health and Nutrition Examination Survey, 1999 to 2004. J Am Coll Surg. 2008; 207(6):928-34. doi: 10.1016/j.jamcollsurg.2008.08.022

7. Manejo intrahospitalario de la diabetes tipo 2. En: Revista de la Asociación Latinoamericana de Diabetes. Guías ALAD sobre diagnóstico, control y tratamiento de la diabetes mellitus tipo 2 con medicina basada en la evidencia. Edición 2013. ALAD; 2013: p.93-4.

8. Garrido J, Chacón J, Sandoval D, Muñoz M, López N, Oyarzún E, et al. Control del hipertenso, un desafío no resuelto. Avances logrados en Chile mediante el programa de salud cardiovascular. Rev Chil Cardiol. 2013;32(2):85-96.

9. Schargrodsky H, Hernández-Hernández R, Champagne BM, Silva H, Vinueza R, Silva Ayçaquer LC, et al. CARMELA: assessment of the CV risk in seven Latin American cities. Am J Med. 2008;121(1): 58-65. doi: 10.1016/j.amjmed.2007. 08.038

10. De la Sierra A. La monitorización ambulatoria de la presión arterial es un instrumento aconsejable para todos los pacientes. Hipertens Riesgo Vasc. 2017;34(1): 45-99. doi:10.1016/j.hipert.2016.06.004

11. Doménech Feria-Carot M, Sobrino Martínez J. Hipertensión nocturna. Hipertens Riesgo Vasc. 2011; 28(4):143-8. doi:10.1016/j.hipert.2011.03.005

12. Turnbull F; Blood Pressure Lowering Treatment Trialists Collaboration. Effects of different blood-pressure-lowering regimens on major cardiovascular events: results of prospectively-designed overviews of randomised trials. Lancet 2003;362(9395): $1527-35$.

13. Fasce E, Campos I, Ibáñez P, Flores M, Zárate H, Román O, et al. Trends in prevalence, awareness, treatment and control of hypertension in urban communities in Chile. J Hypertens. 2007;25(9):1807-11. doi:10.1097/HJH.0b013e328244e481 